\title{
A NEW APPROACH ON NOISE ESTIMATION OF IMAGES
}

\author{
Mredhula $\mathrm{L}^{1}$, Dorairangaswamy $\mathrm{M} \mathrm{A}^{2}$ \\ ${ }^{I}$ Research scholar, Department of CSE, Sathyabama University, Tamil Nadu, India \\ ${ }^{2}$ Prof \& Head, Department of CSE \&IT, AVIT, Chennai, Tamil Nadu, India
}

\begin{abstract}
This paper proposes a new idea towards the researches on noise estimation on images. Image Denoising is always a challenging field as visual quality factor plays an important role. The noise estimation process is done in the transformed domain. In this work, Wavelet Transform is used because of its sparse nature. Then a Bayesian Approach is adopted by imposing an a priori Gaussian Distribution on the transformed pixels. The image quality is checked before entering to noise estimation process by using Maximum-Likelihood Decision criterion. Then a new bound based estimation process is designed by taking the idea from Cramer-Rao Lower Bound for signals in Additive White Gaussian Noise. A visually better result is observed in the experimental output which is obtained after reconstructing the original image.
\end{abstract}

Keywords: Image Denoising; Wavelet Transform; Bayesian Approach; Gaussian Distribution; Maximum-Likelihood Decision Criterion, Cramer-Rao Lower bound

\section{INTRODUCTION}

Image Denoising is a wide area which is aiming for a dream goal of all researchers to make 99 percentage signal estimation accuracy. An image is corrupted by various kinds of noises in its transmission. The main aim of image denoising is to obtain the original image from noisy image. Attempts to remove noise may introduce artifacts and may also cause blurring of the images.

Image restoration is a method of removal or reduction of degradation that occurs during the image capturing. Degradation comes from blurring as well as noise due to the electronic and photometric sources. Due to relative motion between camera and original scene or may be due to misfocus of the cameras blurring may occur. Any unwanted signal that interfers with the original image will degrade the visual quality of the image. The main sources of noise in digital images are imperfect instruments, problem with data acquisition process, transmission and compression [1]. Denoising of Images forms the pre-processing step in the field of research, technology and medical science, where image has been degraded and restoring has to be done before further processing.

Most of the recent research on image denoising has been focused on reducing noise in transform domain. Starting with work of Donoho, many of the denoising techniques are in wavelet transform domain. In the sliding-window transform, the basic idea is to successively denoise overlapping blocks by doing the coefficient shrinkage in local 2D transform domain (e.g. DCT, DFT, etc.). The transform-based approaches give very good overall performance in terms of objective criteria, but they fail to preserve details and may often introduce artifacts that are characteristic of this transform. Unlike the transform-based ones, the spatial methods introduce very few artifacts in the estimates but often over smooth image details. The concept of employing similar data patches from different locations is popular in the video processing field where noise is attenuated in 3D DCT domain under the term of blockmatching. Traditionally, block-matching has found successful application in conjunction with transform-based techniques. [2]. Thresholding and Bayesian modeling are major classification for recent studies. Among these, Bayesian level denoising performs well compared to soft thresholding techniques. The four main image models used for denoising are the Markovian-Bayesian paradigm, linear transform thresholding, image sparsity, and an image selfsimilarity hypothesis [3], [4], [5]. The first modelling is related to some probabilistic ideas. A probability density function is derived and parameter estimation is done. In the second modelling, thresholding of the coefficients is done. All coefficients that are lower than the threshold are modified. In the third type of modelling, sparse natures of image is exploited and in the final modelling Blockiness effect of pixels are arranged.

\section{WAVELET AS A TARNSFORMATION TOOL}

The first use of wavelets was by Haar in 1909. He was interested in finding a basis on a functional space similar to Fourier's basis in frequency space. In physics, wavelets were used in the characterization of Brownian motion. This work led to some of the ideas used to construct wavelet bases. In the signal processing field, S. Mallat discovered the filter banks which have important connections with wavelet basis function. Compression is the area of application of Wavelet in the Signal Processing application. If we can create a suitable representation of a signal, by discarding the least significant pieces of that representation and the original signal can be kept intact. For this a transformation may be required which separates the important parts of the signal from less important parts [6]. 
In simple, it is possible to decompose a signal into two parts: a low frequency part and a high frequency part. The low frequency part may be some sort of average of the original signal and is smoother representation of the original image, and high frequency part, is what we get when the low frequency part is subtracted from the original signal. All Wavelets commonly use this approach that of decomposing a signal into two parts. Also fundamental to wavelet analysis is a hierarchical decomposition, in which we may apply further transforms to an already decomposed signal [6]. In this work, high frequency coefficients form the set of interest. Whole estimation process is done in this region. Also the coefficient set must be compact and thus the transformation must be having sparse nature. This is the reason behind the selection of wavelet as a transformation tool. Those high frequency noise coefficients are efficiently removed by applying a new statistical based estimation approach.

\section{BAYESIAN CONCEPT ON TRANSFORMED PIXEL}

Bayesian approach is a prior modeling of wavelet coefficients without having the true knowledge of the noise being added. In effect it is argued that priors don't require any subjective input. There are various prior choices taken from estimation theory. A popular one is the Generalized Gaussian Distribution (GGD) or the $\alpha$-stable prior. . Other modes of distributions accepted are also of Gaussian nature, they are $\alpha$-stable distribution and Bessel $\mathrm{K}$ Form distribution. Any kind of information sets is assumed to have a Gaussian nature. One of the main disadvantages of GGD is that it fails to capture the tail coefficient measure. The $\alpha$-stable prior can clear this fault of GGD by capturing the tail behavior. But their estimator is poor in the presence of high noise, providing low PSNR values[13]. The third prior distribution model is called Bessel K Forms (BKF). It has more advantage compared to the other two. It shows the unique feature of kurtosis[7],[8].

Here it's assumed that the pixels follow Gaussian Distribution with $\mu$ mean and $\sigma$ variance. A general Probability Density Function for a random variable $\mathrm{x}$ is defined as follows:

\section{DETECTION OF PRESENCE OF TRUE PIXEL}

Detecting the presence of signal by framing a decision rule [9] is considered as an intermediate step. There are some decision criterions which are applied in signals and distributions. In decision theory, the main step of decision making is done with a minimum percentage of uncertainty. Most simple decision making step is Maximum-Likelihood decision criterion. In this decision rule the consideration is only to the most likely event. Other decision rule is Neyman-Pearson criterion. It says that a decision rule is designed to get maximum probability of detection while not allowing the error probability of false alarm to exceed a certain value alpha The Probability of error criterion says to select the decision regions so as to minimize the total probability of error (probability to make an incorrect decision). Bayes Risk criterion employs a systematic procedure of assigning a cost value to each correct and incorrect decision and evaluating total cost. This total average cost is to be minimized. There are some cases in which the a priori probabilities of messages may not be known. In such cases Min-Max criterion is used [9]. This is a modified version of previous method. MaximumLikelihood Decision Criterion is used for the implementation of this step in this work. Criterion is depicted as:

- $\mathrm{m} 1: \mathrm{X}=\mathrm{n}$

- $\mathrm{m} 2: \mathrm{X}=$ image + noise

- Conditional probabilities are $\mathrm{P}(\mathrm{X} / \mathrm{m} 1)$ and $\mathrm{P}(\mathrm{X} / \mathrm{m} 2)$

- $\quad$ Likelihood Ratio:: $\Lambda(X)=\mathrm{P}(\mathrm{X} / \mathrm{m} 2) / \mathrm{P}(\mathrm{X} / \mathrm{m} 1)$

- Decision::

$$
\Lambda(\mathrm{X})>1 \text {----d2(Corresponding to } \mathrm{m} 2 \text { ) }
$$

$\Lambda(\mathrm{X})<1-----\mathrm{d} 1$ (Corresponding to $\mathrm{m} 1$ )

where $\mathrm{m} 1$ and $\mathrm{m} 2$ are the two messages with which a likelihood ratio is calculated. After this step, a decision rule is imposed as per the criterion. This leads to an approximate range for the unknown variable (variable is taken as the pixel levels/gray levels for an image). That maximum range with positive value is selected as the detection threshold. If a max no. of gray levels exceed this threshold value (intensity level for an image), which is the detection threshold, then that image is selected for further estimation.

\section{A NEW APPROACH FOR NOISE}

\section{ESTIMATION}

In general, detection and estimation applications involve making inferences from observations that distorted or corrupted in some manner. As the information that one wish to extract from such observation is unknown to the observer, it is useful to cast detection and estimation problems in a probabilistic framework in which unknown behavior is assumed to be random.

The estimation and parameterization methods are mainly by several iterative algorithms. Two algorithm used are Belief Propagation (BP) algorithm and Expectation Maximization (EM) algorithm. In Expectation Maximization (EM) algorithm, the EM iteration alternates between performing an expectation (E) step, in which a function is created for the expectation of the log-likelihood evaluated using the current estimate for the parameters, and maximization (M) step, which computes parameters maximizing the expected log-likelihood found on the E step. These parameterestimates are then used to determine the distribution of the latent variables in the next E step [11]. This works good only if the amount of noise is less and the set dimensionality is small.

Belief propagation is a message passing algorithm which calculates the marginal distribution for each unobserved node, conditional on any observed nodes [12] used in Bayesian networks and Markov random fields. Main 
problem involved in these analyses is the selection of an initial value. The process begins by randomly selecting the initial estimates of the parameters. This leads to the accumulation of error while advancing iterations. Iterations also make the computations more.

This problem of accumulation of error is taken as the main objective of this work. It is argued that other statistical means will minimize this error. Initially selecting a parameter which depends on the noise value and a minimum lower bound is calculated. This bound is the decision parameter for the parameter estimation and thus identifying noise values.

The estimation idea is taken from the famous bound based estimator accuracy argumentation of Cramer-Rao Lower Bound. This bound is calculated for the selected parameter of the pixel distribution. Variance is the selected parameter and it is considered as a vector parameter, i.e . Image is divided into different sub-images and variance levels are found [13]. Estimating a vector parameter where $\sigma \mathrm{s}$ are variances of different sublevels of an image. The CRLB is found as the [i,i] element of the inverse of the matrix, an sxs Fisher Information Matrix [13],

The latter is defined by

for $\mathrm{i}=1,2,3 \ldots \mathrm{s}, \mathrm{j}=1,2,3 \ldots \mathrm{s}$. While evaluating the equation, the true value of $\sigma$ is used. In this work the processing is done pixel by pixel. Therefore the expectation value is not considered while deriving the Fisher Information matrix. This matrix is a covariance score of the selected observation set. Here in this work it's the set of transformed pixels.

\section{EXPERIMENTAL RESULTS AND DISCUSSIONS}

Newly proposed method is experimented on Grey scale Woman image and colour mandrill image. Two experimental sections were detection and estimation processes. Whole processing is done on transformed domain. Selected image is first transformed to wavelet domain. In this experiment, four level wavelet decomposition is employed. Higher level decompositions can be done to extract the minor details. Then an intermediate step is introduced to check whether the received image is qualified for estimation. The discrete wavelet transformed coefficients are modelled as a Gaussian pdf (probability density function) with mean, variance and peak as the parameters. Instead of vector analysis, variance is taken as a scalar for the initial execution. It is observed that the sharpness of the peak decreases as the variance measure increases. A detection threshold is calculated and signal presence is detected using Maximum likelihood Decision Criterion. It's checked that if the received signal contains a maximum of valid signal content. Thus a decision threshold is calculated by applying Maximum-Likelihood decision rule. For the loaded woman image decision threshold obtained as 32.47. Each pixel of the image is compared with this decision threshold value. Threshold value is noted as the pixel level range. As many of the pixels of the image satisfy the decision threshold, then the image is selected for further estimation process.

In the estimation process, the steps to derive Fisher Information Matrix are mathematically coded in Matlab. Conditions are checked for the variance level of the entire image. For the comparison, inverse of Fisher Information matrix is derived. Then the final step of reconstruction is done by doing a reverse estimation process. After doing this ,modified high frequency coefficients are obtained. Finally, the low frequency filtered components of first level wavelet decomposition is added. Thus sufficient information contained in the image is maintained. Obtained results are shown in figure below. The observations are on a gray scale image (Fig-1) and for RGB (Fig-2)

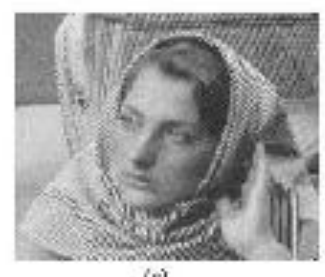

(ei)

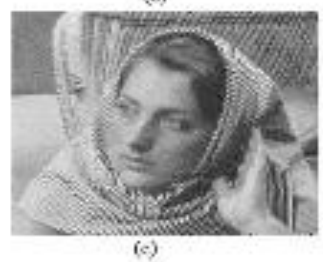

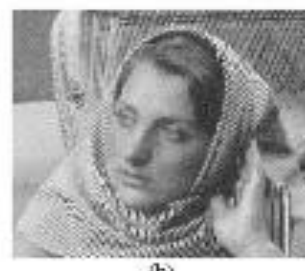

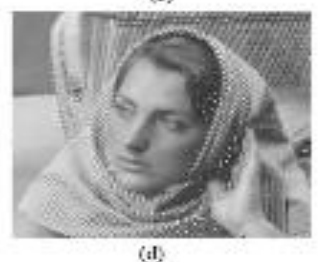

Fig-1 (a) Original Image (b) Noisy Image (c) Sub Image (d) Reconstructed Image

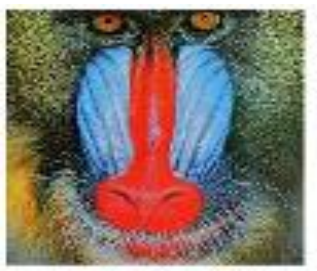

(a)

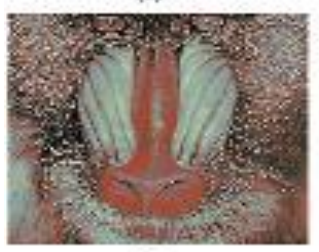

(c)

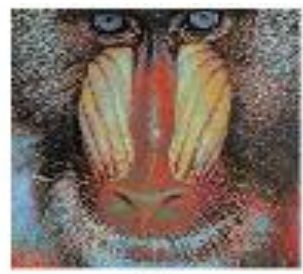

(b)

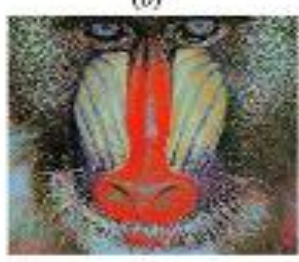

(d)
Fig-2 (a) Original Image (b) Noisy Image (c) Sub Image (d) Reconstructed Image

\section{CONCLUSION AND FUTURE SCOPE}

This work argues that finding an accurate model for the coefficients is the most tedious task in all denoising projects. If it is done, then it is easy to represent the sparse behavior of the wavelet coefficients for a large class of images. The true pixel presence detection process improves the validity of denoising implementation. The result also provides a good match between observed and estimated prior densities. 
Table -1: PSNR VALUES

\begin{tabular}{|l|l|}
\hline WOMAN IMAGE & 33.976 \\
\hline MANDRILL IMAGE & 33.596 \\
\hline
\end{tabular}

The main content of this paper is an application of a new noise estimation method based on statistical means. Whole Estimation based processing is concentrated on high frequency coefficients. It is assumed that noise is accumulated in high frequency bands. Therefore, noise estimation is done in this high frequency domain while preserving information pixels. In the first step of detection process, the image quality is checked. The resultant image matrix is modified by removing all those variance levels which exceed minimum image variance according to the Cramer Rao Lower Bound relation. Then a reverse estimation is done. Final reconstructed image by embedding all image information gave a visually better image which has less noise. It is observed that blur noise is efficiently removed in this method for the selected image.

Here it is assumed that White Gaussian Noise is added up. If the noise cannot be approximated to AWGN and the noise coefficients are correlated, then these estimators cannot be used. The genuinity of this method will be more valued in image processing applications if the processing is done in sub-band levels. The image is to be divided into different sub bands and processing can be done more effectively on each sub bands. The basis of coefficients must be selected after an optimization process. For this process a sub band dependent cost function is to be defined. According to the value of cost function an Optimal Wavelet Basis is selected. This will improve the decisions for detection of the true coefficients. Also the detection threshold value selected must be more optimal. These are the expected modifications of this work.

\section{REFERENCES}

[1]. Vikas Gupta, Vijayshree Chaurasia, Madhu Shandilya, 'A Review on Image Denoising Techniques', International Journal of Emerging Technologies in Computational and Applied Sciences(IJETCAS), 5(2) June-August 2013, pp. 204- 208.

[2]. Kostadin Dabov, Alessandro Foi, Vladimir Katkovnik, and Karen Egiazarian, 'Image denoising with blockmatching and 3D filtering',SPIE, Vol. 6064, 2006.

[3]. Larbi Boubchir and Boualem Boashash, 'Wavelet denoising based on the MAP estimation using the BKF prior with application to images and EEG signals',IEEE Journal of Latex Class Files, Vol. 11, No. 4, December 2012.

[4]. Abdolhossein Fathi and Ahmad Reza Naghsh-Nilchi,' Efficient Image Denoising Method Based on a New Adaptive Wavelet Packet Thresholding Function', IEEE Transanction on Image Processing, vol. 21,no.9,September2012.

[5]. Jalal M. Fadili and Larbi Boubchir, 'Analytical Form for a Bayesian Wavelet Estimator of Images Using the Bessel K Form Densities', IEEE Transactions On Image Processing, Vol. 14, No. 2, February 2005.
[6]. Stephane G. Mallat,' A Theory for Multiresolution Signal Decomposition:The Wavelet Representation', IEEE Transactions On Pattern Analysis And Machine Intelligence, Vol I I , No. 7. July 1989.

[7]. M. Bicego, D. Gonzalez-Jimenez, E. Grosso, J.L. Alba Castro,' Generalized Gaussian Distributions for Sequential Data Classification', ICPR papers, 2008

[8]. Jalal M. Fadili and Larbi Boubchir, 'Analytical Form for a Bayesian Wavelet Estimator of Images Using the Bessel K Form Densities', IEEE Transactions On Image Processing, Vol. 14, No. 2, February 2005.

[9]. James L Melsa and David L Cohn, a textbook on 'Detection and Estimation Theory', University of Notre Dame, McGraw-Hill, 1978

[10]. Priyam Chatterjee and Peyman Milanfar, 'Is Denoising Dead?', IEEE Transactions on Image Processing, Vol.19, No.4, April 2010.

[11]. Ajit Singh,'EM Algorithm', November 20,2005 